\title{
Suppression of Repeat-Intensive False Targets Based on Temporal Pulse Diversity
}

\author{
Gang Lu, ${ }^{1}$ Yongqiang Chen, ${ }^{1}$ Yu Lei, ${ }^{1}$ and Guan Gui ${ }^{2}$ \\ ${ }^{1}$ School of Electrical and Information Engineering, Xihua University, Chengdu 610039, China \\ ${ }^{2}$ Department of Communications Engineering, Graduate School of Engineering, Tohoku University, Sendai 980-8579, Japan
}

Correspondence should be addressed to Gang Lu; aeneas999@hotmail.com

Received 8 July 2013; Accepted 11 September 2013

Academic Editor: Mandeep Singh Jit Singh

Copyright (c) 2013 Gang Lu et al. This is an open access article distributed under the Creative Commons Attribution License, which permits unrestricted use, distribution, and reproduction in any medium, provided the original work is properly cited.

\begin{abstract}
This paper considers the problem of suppressing the repeat-intensive false targets produced by a deception electronic attack (EA) system equipped with a Digital Radio Frequency Memory (DRFM) device. Different from a conventional repeat jammer, this type of jamming intensively retransmits the intercepted signal stored in a DRFM to the victim radar in a very short time-delay interval relative to a radar pulse wide. A multipeak matched-filtering output is then produced other than the merely expected true target. An electronic protection (EP) algorithm based on the space time block code (STBC) is proposed to suppress the adverse effects of this jammer. By transmitting a pulse sequence generated from the STBC in succession and the following cancellation process applied upon the received signal, this algorithm performs successfully in a single antenna system provided that the target models are nonfluctuating or slow fluctuating and the pulse repetition frequency (PRF) is comparatively high. The performance in white and correlated Gaussian disturbance is evaluated by means of Monte Carlo simulations.
\end{abstract}

\section{Introduction}

In electronic warfare (EW) area, the false target is a common deception electronic attack (EA) method employed to counter against radar systems [1-3]. However, just repeating the intercepted radar signal, as done in the conventional repeat jammer, is not effective because only one false target is produced. The jamming type of repeat-intensive false targets is presented in recent researches, and it can produce high-intensive matched-filter output peaks which are similar to the true target and commonly have power higher than the true one. Moreover, based on the Digital Radio Frequency Memory (DRFM) device, these false targets generally have all the qualities typical of the true target specified. It is difficult for the radar to detect and discriminate the true target from a multitude of spikes which are positioned ahead or behind the true target and spaced closer than the time duration of the radar backscattering signal.

The pulse diversity-based approach is a powerful electronic protection (EP) to counter multiform jammers [4, 5]. Based on varying the radar chirp rate or utilizing a phase modulated signal for phase perturbations in a linear frequency modulated (LFM) signal, Soumekh [4] used penalization measures to reduce the power of a conventional DRFM jammer in synthetic aperture radar. Another signalling scheme is proposed to combat the impact of repeat jammers by continuously emitting modified version of previous waveforms or a new waveform following a specific orthogonal structure [5]. And that, if orthogonal signal in sequence is permitted, the jammer can be rejected or mitigated by transmitting signals orthogonal to each other.

On the other hand, the mentioned approaches do not involve the repeat-intensive false targets jammer, but the conventional one. Generally, the concept of diversity can be used to reduce multipath effect. Moreover, the diversity of frequency, space, time, and so forth is successfully applied in space-time adaptive processing (STAP) or multiple-input multiple-output (MIMO) system to reduce channel fading, suppress interferences or clutters, and improve system performance [6-9].

Here, we employ a space time block code (STBC) to counter against the repeat-intensive false targets jammer. In our work, however, we focus on a single antenna system. 
The rest of this paper is organized as follows. In Section 2, the repeat-intensive false targets are briefly introduced. The signal models, as well as the concept of algorithm, are developed in Section 3 based on STBC by using temporal pulse diversity scheme specifically. Numerical performance simulations and analysis are described in Section 4. Finally, conclusions are drawn in Section 5.

\section{Repeat-Intensive False Targets}

The jammer of repeat-intensive false targets originates from the common DRFM false target. By repeating an intercepted radar data recorded in a DRFM device, a common false target jammer produces one target which is not really existent to protect the true target. And, an improved version of it, the jammer of multifalse targets, provides an enhanced jamming effect by yielding more than one imaginary targets dispersed along the ranges in victim radar side. If the number of produced target is large and the interval between every two leading edge of each pulse is short (compared with a pulse duration), high dense of false targets can be generated and the desired echoes will be suppressed in view of the positive jammer-to-signal ratio (JSR). This is referred to as repeatintensive false targets jammer, where the signal interval of compressed narrow spike is short relative to the original radar pulse duration.

It is assumed that radar signals are intercepted by a hostile ECM equipment. At the time of repeating, the data stored in a DRFM are read and then converted from a digital signal into an analog signal with a digital-to-analog converter. Subsequently, the analog signal is upconverted in frequency and transmitted back to the victim radar. After a time-delay interval, the data are read again from the initial address and added to the former data (untransmitted segments), and then they were manipulated in the same way until the predestined number of false target is reached (or transmitted circularly). Thus the produced repeat-intensive false targets can be expressed as

$$
\mathbf{j}(t)=\sum_{l} \rho_{l} \mathbf{j}_{s}\left(t-\tau_{l}\right)
$$

where $\mathbf{j}_{s}(t)$ denotes the column vector of the intercepted radar signal and $\rho_{l}$ and $\tau_{l}$ are the complex amplitude and time-delay interval of each subjamming signal, respectively.

\section{Algorithm Formulation}

3.1. Signal Models. We assume here that the complex vector recorded by the radar during the time on target (ToT) is as follows:

$$
\mathbf{z}=\left[\begin{array}{llll}
z(0) & z(1) & \cdots & z(K)
\end{array}\right]^{T},
$$

where $K$ is the number of samples during a single pulse.

It is assumed that the recorded samples consist of the sum of the target and the disturbance; that is

$$
\mathbf{z}=p \mathbf{s}+\mathbf{d},
$$

where $\mathbf{s}$ is the signal vector, $p$ is the complex amplitude, d is the Gaussian disturbance which is defined as the sum of white thermal noise $\mathbf{n}$ and correlated Gaussian clutter c, where $\mathbf{n} \sim \mathrm{CN}\left(0, \sigma_{n}^{2} \mathbf{I}\right), \mathbf{c} \sim \mathrm{CN}\left(0, \sigma_{c}^{2} \mathbf{R}\right)$, and $\mathbf{I}$ and $\mathbf{R}$ are the identity matrix and normalized clutter covariance matrix, respectively. Consequently, $\mathbf{d}$ is modeled as complex Gaussian distributed random vector with zero mean and covariance matrix $\sigma_{d}^{2} \mathbf{M}=E\left(\mathbf{z z}^{H}\right)$, where $\sigma_{d}^{2}=\sigma_{n}^{2}+\sigma_{c}^{2}$ is the total disturbance power and $\mathbf{M}$ is the normalized disturbance covariance matrix formulated as follows:

$$
\mathbf{M}=\frac{\sigma_{n}^{2} \mathbf{I}+\sigma_{c}^{2} \mathbf{R}}{\sigma_{n}^{2}+\sigma_{c}^{2}}=\frac{1}{\mathrm{CNR}+1} \mathbf{I}+\frac{\mathrm{CNR}}{\mathrm{CNR}+1} \mathbf{R} .
$$

Denote by CNR the clutter-to-noise power ratio, more precisely, $\mathrm{CNR}=\sigma_{c}^{2} / \sigma_{n}^{2}$. In shorthand notation, $\mathbf{d} \sim \mathrm{CN}\left(0, \sigma_{d}^{2} \mathbf{M}\right)$. Furthermore, we suppose that a set of secondary data free of signal components but sharing the same statistical properties of the disturbance in the cell under test is available.

Here, $\mathbf{M}$ is Hermitian, and we assume that $\mathbf{M}$ is full rank also (this condition is almost satisfied in practice). Therefore, it can be factored as $\mathbf{M}=\mathbf{L} \mathbf{L}^{H}$, where $\mathbf{L}$ is a lower triangular matrix. The output of the whitening-matched filter (WMF) $[10]$ is given by

$$
\begin{aligned}
F(\mathbf{z}) & =\mathbf{s}^{H} \mathbf{M}^{-1} \mathbf{z}=\mathbf{z}^{H} \mathbf{M}^{-1} \mathbf{s} \\
& =\left(\mathbf{L}^{-1} \mathbf{z}\right)^{H}\left(\mathbf{L}^{-1} \mathbf{s}\right) .
\end{aligned}
$$

In this formulation, $\mathbf{L}^{-1} \mathbf{z}$ denotes the new whitening received vector, while $\mathbf{L}^{-1} \mathbf{s}$ denotes the new whitening transmitting signal replica of radar. If the filter realization form is used, the corresponding impulse response of the $\mathrm{WMF}$ is $\mathbf{h}=\mathbf{s}$. Then at time $t$, the whitening matched-filtering output is given by $y(t)=\mathbf{h}^{H} \mathbf{M}^{-1} \mathbf{z}(t)$, where $\mathbf{M}^{-1} \mathbf{z}(t)$ denotes the transformed input signal vector at time $t$.

3.2. STBC Pulse Sequence. If multiple antennas are available, it is convenient to implement space diversity. In our algorithm, however, a STBC is applied in sequence (temporally); therefore we focus on a single antenna system.

We formulate the suppression approach as a temporal pulse diversity problem on the STBC Q [11]:

$$
\mathbf{Q}=\sum_{m=1}^{M}\left(\mathbf{x}_{m} \mathbf{A}_{m}+\mathbf{x}_{m}^{*} \mathbf{B}_{m}\right)
$$

where $\mathbf{A}_{m}$ and $\mathbf{B}_{m}$ are $N \times N_{t}$ matrices and $\left\{\mathbf{x}_{m}\right\}_{m=1}^{M}$ is the transmission signal set. Particularly, $\mathbf{Q}$ is also referred to as Alamouti code [12] if $N=N_{t}=M=2$ and the following expressions are satisfied:

$$
\begin{array}{ll}
A_{1}=\left[\begin{array}{ll}
1 & 0 \\
0 & 0
\end{array}\right], & A_{2}=\left[\begin{array}{ll}
0 & 1 \\
0 & 0
\end{array}\right], \\
B_{1}=\left[\begin{array}{ll}
0 & 0 \\
0 & 1
\end{array}\right], & B_{2}=\left[\begin{array}{cc}
0 & 0 \\
-1 & 0
\end{array}\right] .
\end{array}
$$

We use this scheme in view of that $N=N_{t}=M=2$ is the minimum order block code. Certainly, the higher-order 
block codes can be handled in the same way and may provide further features $[13,14]$.

Suppose that the radar transmission pulses in the first 4pulse repetition intervals (PRI) are given by

$$
\mathbf{s}_{T}=\left[\begin{array}{llll}
\mathbf{s}_{1}^{T}(t) & \mathbf{s}_{2}^{T}(t) & \mathbf{s}_{3}^{T}(t) & \mathbf{s}_{4}^{T}(t)
\end{array}\right]^{T},
$$

where $\mathbf{s}_{i}(t), i=1$ to 4 , denotes the radar transmission signal vector in sequence PRI.

When radar signals are received by a jammer, the jammer needs to detect and analyze the incoming pulses in order to handle the valid signals and produce effective jamming pulses. It requires a certain amount of time to accomplish the whole process with the updated pulse $[4,5]$. It is then possible for the radar to operate with the jamming signal recorded in previous PRI. Moreover, even if a jammer could detect and retransmit the current pulse at once, it would have to repeat the pulse recorded at previous PRI when it attempts to protect targets which are closer to the victim radar than itself [5]. As a consequence, it is assumed that the jammer lags one PRI behind the victim radar. If the delayed PRI is greater than one, a higher-order STBC can be utilized to overcome this challenge.

Conclusively, the jamming pulses can be expressed as

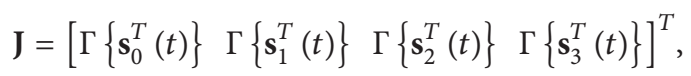

where the bounded linear operator $\Gamma$ denotes the process or transformation performed on the radar signals to produce the repeat-intensive false targets. In the initial pulse diversity block, $\mathbf{s}_{0}(t)$ may be any arbitrary pulse (signal stored in a DRFM), while, on the contrary, it is definite in subsequent blocks, just the last radar transmission pulse.

The received pulse blocks at the radar side can therefore be modeled as follows:

$$
\begin{aligned}
\mathbf{z}_{1}(t)= & \sum_{m} p_{m}(1) \mathbf{s}_{1}\left[t-\tau_{m}(1)\right] \\
& +\sum_{n} p_{n}^{\prime}(1) \Gamma\left\{\mathbf{s}_{0}\left[t-\tau_{n}^{\prime}(1)\right]\right\}+\mathbf{d}_{1}(t), \\
\mathbf{z}_{2}(t)= & \sum_{m} p_{m}(2) \mathbf{s}_{2}\left[t-\tau_{m}(2)\right] \\
& +\sum_{n} p_{n}^{\prime}(2) \Gamma\left\{\mathbf{s}_{1}\left[t-\tau_{n}^{\prime}(2)\right]\right\}+\mathbf{d}_{2}(t), \\
\mathbf{z}_{3}(t)= & \sum_{m} p_{m}(3) \mathbf{s}_{3}\left[t-\tau_{m}(3)\right] \\
& +\sum_{n} p_{n}^{\prime}(3) \Gamma\left\{\mathbf{s}_{2}\left[t-\tau_{n}^{\prime}(3)\right]\right\}+\mathbf{d}_{3}(t), \\
& +\sum_{n} p_{n}^{\prime}(4) \Gamma\left\{\mathbf{s}_{3}\left[t-\tau_{n}^{\prime}(4)\right]\right\}+\mathbf{d}_{4}(t),
\end{aligned}
$$

where $p_{m}(i)$ and $p_{n}^{\prime}(i)(i=1$ to 4$)$ denote the complex amplitude of the target and jamming signal, respectively, $\tau_{m}(i)$ and $\tau_{n}^{\prime}(i)(i=1$ to 4$)$ denote the time delay of the target and jamming signal, respectively, and $\mathbf{d}_{i}(t)(i=1$ to 4$)$ denotes the complex Gaussian disturbance.
3.3. Concept of Jamming Suppression. In this work, the magnitude of the target is assumed to be relatively constant during the dwell time. More precisely, we assume that the targets are nonfluctuating or slow fluctuating. Therefore, $p_{m}(i)$ and $p_{n}^{\prime}(i)$ in (10) can be replaced succinctly by $p_{m}$ and $p_{n}^{\prime}$, respectively.

Under the condition of static state, it is clear that $\tau_{m}(i)=$ $\tau_{m}$ and $\tau_{n}^{\prime}(i)=\tau_{n}^{\prime}$ for $i=1$ to 4 . Otherwise, if this condition is not satisfied, the same conclusion can be drawn in most practical cases provided that pulse repetition frequency (PRF) is comparatively high. In order to explain it, we first model the round-trip delay $\tau_{m}(i)$ as $\tau(t)=2(R-v t) / c$, where $R$ is the initial range of target, $v$ is the average radial velocity of target, and $c$ is the velocity of light. Actually, the magnitude of time-delay difference in a pulse block is very small, generally in the level of nanosecond. As we will see later, the pulses in intervals 2 and 4 (or intervals 1 and 3 ) in one block are picked out to process together in order to suppress the false target. The corresponding time-delay difference is then $\Delta \tau=4 v /\left(c f_{p}\right)$, where $f_{p}$ denotes PRF. Here, because $v$ is much smaller than $c$ and $f_{p}$ is comparatively high just as we assumed, the errors introduced by the time-delay difference are less than one sampling point for narrow band radars in most practical cases. If this condition is not satisfied in some exceptional cases, that is, the time-delay difference cannot be neglected, we can compensate it by a delay factor when it can be estimated or known as a priori. Due to similar reasons (except the single-trip), the same conclusion can be drawn for $\tau_{n}^{\prime}(i)$. Therefore, $\tau_{m}(i)$ and $\tau_{n}^{\prime}(i)$ can be replaced by $\tau_{m}$ and $\tau_{n}^{\prime}$, respectively. Then, (10) can be expressed in the following form:

$$
\begin{aligned}
\mathbf{Z}=\left[\begin{array}{ll}
\sum_{m} \mathbf{s}_{1}\left(t-\tau_{m}\right) & \sum_{n} \Gamma\left\{\mathbf{s}_{0}\left(t-\tau_{n}^{\prime}\right)\right\} \\
\sum_{m} \mathbf{s}_{2}\left(t-\tau_{m}\right) & \sum_{n} \Gamma\left\{\mathbf{s}_{1}\left(t-\tau_{n}^{\prime}\right)\right\} \\
\sum_{m}^{n} \mathbf{s}_{3}\left(t-\tau_{m}\right) & \sum_{n} \Gamma\left\{\mathbf{s}_{2}\left(t-\tau_{n}^{\prime}\right)\right\} \\
\sum_{m} \mathbf{s}_{4}\left(t-\tau_{m}\right) & \sum_{n} \Gamma\left\{\mathbf{s}_{3}\left(t-\tau_{n}^{\prime}\right)\right\}
\end{array}\right]\left[\begin{array}{c}
p_{m} \\
p_{n}^{\prime}
\end{array}\right] \\
+\left[\begin{array}{l}
\mathbf{d}_{1}(t) \\
\mathbf{d}_{2}(t) \\
\mathbf{d}_{3}(t) \\
\mathbf{d}_{4}(t)
\end{array}\right],
\end{aligned}
$$

where $\mathbf{Z}=\left[\begin{array}{llll}\mathbf{z}_{1}^{T}(t) & \mathbf{z}_{2}^{T}(t) & \mathbf{z}_{3}^{T}(t) & \mathbf{z}_{4}^{T}(t)\end{array}\right]^{T}$.

In order to suppress the repeat-intensive false targets, we resort to the second-order STBC here; that is, inserting (7) and $N=N_{t}=M=2$ into (6), we obtain the transmission pulses:

$$
\begin{aligned}
\mathbf{S}_{T} & =\left[\begin{array}{llll}
\mathbf{s}_{1}^{T}(t) & \mathbf{s}_{2}^{T}(t) & \mathbf{s}_{3}^{T}(t) & \mathbf{s}_{4}^{T}(t)
\end{array}\right]^{T} \\
& =\left[\begin{array}{llll}
\mathbf{x}_{1}^{T}(t) & \mathbf{x}_{2}^{H}(t) & -\mathbf{x}_{2}^{T}(t) & \mathbf{x}_{1}^{H}(t)
\end{array}\right]^{T} .
\end{aligned}
$$


Then (11) can be recast as

$$
\begin{aligned}
& \mathbf{Z}= {\left[\begin{array}{cc}
\sum_{m} \mathbf{x}_{1}\left(t-\tau_{m}\right) & \sum_{n} \Gamma\left\{\mathbf{x}_{0}\left(t-\tau_{n}^{\prime}\right)\right\} \\
\sum_{m} \mathbf{x}_{2}^{*}\left(t-\tau_{m}\right) & \sum_{n} \Gamma\left\{\mathbf{x}_{1}\left(t-\tau_{n}^{\prime}\right)\right\} \\
-\sum_{m} \mathbf{x}_{2}\left(t-\tau_{m}\right) & \sum_{n} \Gamma\left\{\mathbf{x}_{2}^{*}\left(t-\tau_{n}^{\prime}\right)\right\} \\
\sum_{m} \mathbf{x}_{1}^{*}\left(t-\tau_{m}\right) & -\sum_{n} \Gamma\left\{\mathbf{x}_{2}\left(t-\tau_{n}^{\prime}\right)\right\}
\end{array}\right]\left[\begin{array}{c}
p_{m} \\
p_{n}^{\prime}
\end{array}\right] } \\
&+\left[\begin{array}{l}
\mathbf{d}_{1}(t) \\
\mathbf{d}_{2}(t) \\
\mathbf{d}_{3}(t) \\
\mathbf{d}_{4}(t)
\end{array}\right] .
\end{aligned}
$$

It is convenient to reshape the vectors $\mathbf{Z}$ and $\mathbf{S}_{T}$ into matrices $\widehat{\mathbf{Z}}$ and $\widehat{\mathbf{S}}_{T}$, respectively; that is, $\widehat{\mathbf{Z}}=$ $\left[\begin{array}{llll}\mathbf{z}_{1}(t) & \mathbf{z}_{2}(t) & \mathbf{z}_{3}(t) & \mathbf{z}_{4}(t)\end{array}\right]$ and $\widehat{\mathbf{S}}_{T}=\left[\begin{array}{llll}\mathbf{s}_{1}(t) & \mathbf{s}_{2}(t) & \mathbf{s}_{3}(t) & \mathbf{s}_{4}(t)\end{array}\right]$. As a result, we can use a WMF to handle the received data, more precisely:

$$
\mathbf{Y}=\widehat{\mathbf{S}}_{T}^{H} \mathbf{M}^{-1} \widehat{\mathbf{Z}}
$$

Actually, the diagonal elements of matrix Y denote the output with regard to the true target. In consequence, the 2 nd and 4th diagonal elements, which are corresponding to the pulses in intervals 2 and 4, are picked out to suppress the false targets and focus on the true targets (actually the 1st and 3rd elements can play the same role, but other elements are not necessary for this purpose). More precisely, the filter outputs in intervals 2 and 4 with respect to the true targets are given in detail by

$$
\begin{aligned}
F\left[\mathbf{z}_{2}(t)\right]= & \mathbf{s}_{2}^{H} \mathbf{M}^{-1} \mathbf{z}_{2}(t) \\
= & \sum_{m} p_{m} F\left[\mathbf{x}_{2}^{*}\left(t-\tau_{m}\right)\right] \\
& +\sum_{n} p_{n}^{\prime} \mathbf{x}_{2}^{T}(t) \mathbf{M}^{-1} \Gamma\left\{\mathbf{x}_{1}\left(t-\tau_{n}^{\prime}\right)\right\} \\
& +\mathbf{x}_{2}^{T}(t) \mathbf{M}^{-1} \mathbf{d}_{2}(t), \\
F\left[\mathbf{z}_{4}(t)\right]= & \mathbf{s}_{4}^{H} \mathbf{M}^{-1} \mathbf{z}_{4}(t) \\
= & \sum_{m} p_{m} F\left[\mathbf{x}_{1}^{*}\left(t-\tau_{m}\right)\right] \\
& -\sum_{n} p_{n}^{\prime} \mathbf{x}_{1}^{T}(t) \mathbf{M}^{-1} \Gamma\left\{\mathbf{x}_{2}\left(t-\tau_{n}^{\prime}\right)\right\} \\
& +\mathbf{x}_{1}^{T}(t) \mathbf{M}^{-1} \mathbf{d}_{4}(t) .
\end{aligned}
$$

Denote by $\sum_{m} p_{m} F\left[\mathbf{x}_{2}^{*}\left(t-\tau_{m}\right)\right]$ and $\sum_{m} p_{m} F\left[\mathbf{x}_{1}^{*}\left(t-\tau_{m}\right)\right]$ the matched-filtering outputs that contain target echoes of interest. Evidently, the second terms in the right side of (15) and (16), that is, $\sum_{n} p_{n}^{\prime} \mathbf{x}_{2}^{T}(t) \mathbf{M}^{-1} \Gamma\left\{\mathbf{x}_{1}\left(t-\tau_{n}^{\prime}\right)\right\}$ and $\sum_{n} p_{n}^{\prime} \mathbf{x}_{1}^{T}(t) \mathbf{M}^{-1} \Gamma\left\{\mathbf{x}_{2}\left(t-\tau_{n}^{\prime}\right)\right\}$, both relate to the jammer. In view of $(1)$, it is clear that $\mathbf{j}(t)=\Gamma\left\{\mathbf{x}_{i}(t)\right\}$ and that $\mathbf{j}_{s}(t)=\mathbf{x}_{i}(t)$ here, and this results in $\Gamma\left\{\mathbf{x}_{i}(t)\right\}=\sum_{l} \rho_{l} \mathbf{x}_{i}\left(t-\tau_{l}\right)$. Consequently, we have

$$
\begin{aligned}
& \sum_{n} p_{n}^{\prime} \mathbf{x}_{2}^{T}(t) \mathbf{M}^{-1} \Gamma\left\{\mathbf{x}_{1}\left(t-\tau_{n}^{\prime}\right)\right\} \\
& =\sum_{n} \sum_{l} \rho_{l} p_{n}^{\prime} \mathbf{x}_{2}^{T}(t) \mathbf{M}^{-1} \mathbf{x}_{1}\left(t-\tau_{n}^{\prime}-\tau_{l}\right), \\
& \sum_{n} p_{n}^{\prime} \mathbf{x}_{1}^{T}(t) \mathbf{M}^{-1} \Gamma\left\{\mathbf{x}_{2}\left(t-\tau_{n}^{\prime}\right)\right\} \\
& =\sum_{n} \sum_{l} \rho_{l} p_{n}^{\prime} \mathbf{x}_{1}^{T}(t) \mathbf{M}^{-1} \mathbf{x}_{2}\left(t-\tau_{n}^{\prime}-\tau_{l}\right) .
\end{aligned}
$$

It is easy to verify that, in our case, the jammer-related terms are equal by noting that $\mathbf{M}^{-1}=\left(\mathbf{L L}^{H}\right)^{-1}$. Substitution of (17) and (18) into the sum of (15) and (16) yields

$$
\begin{gathered}
r(t)=\sum_{m} p_{m} F\left[\mathbf{x}_{2}^{*}\left(t-\tau_{m}\right)\right]+\sum_{m} p_{m} F\left[\mathbf{x}_{1}^{*}\left(t-\tau_{m}\right)\right] \\
+\mathbf{x}_{2}^{T}(t) \mathbf{M}^{-1} \mathbf{d}_{2}(t)+\mathbf{x}_{1}^{T}(t) \mathbf{M}^{-1} \mathbf{d}_{4}(t) .
\end{gathered}
$$

It is clear that the jamming terms have been eliminated in (19), and the target echoes are preserved. Therefore, the repeat-intensive false targets can be cancelled out by exploiting the temporal pulse diversity techniques.

\section{Simulations}

As described in Section 3, the jammer-related terms can be cancelled completely if the required conditions are satisfied. No other special constrains have been imposed upon the derivation process.

In order to evaluate the performance of the proposed method, we define the signal-to-jammer-plus-disturbance ratio (SJDR) improvement factor as $I F=S J D R_{2} / S J D R_{1}$, where $S_{1 D R}$ is the input SJDR, while $S_{D D R}$ is the output SJDR after using the algorithm.

In the simulations, $\mathbf{x}_{1}(t)$ is set identical with $\mathbf{x}_{2}(t)$. Here, a LFM signal with bandwidth $5 \mathrm{MHz}$ and time width $10 \mu \mathrm{s}$ and a biphase coded signal with $1 \mathrm{MHz}$ and time width $40 \mu \mathrm{s}$ are utilized as the original radar signals. It is assumed that the target echoes are received in the presence of the repeatintensive false targets jammer. For simplicity, we also assume that the intercepted radar signal in the jammer is repeated for 10 times in uniform time-delay interval. Additionally, we utilize the model $\{\mathbf{M}\}_{i, j}=\sigma_{d}^{2} \rho^{|i-j|}$ concerning the disturbance covariance matrix, and we would investigate the two cases of $\rho=0$ (the Gaussian white disturbance) and $\rho=0.9$ (the Gaussian correlated disturbance).

In Figures 1 and 2, we show the performance IF, for the LFM signal, as a function of the signal-to-disturbance ratio (SDR). The improvement factor IF has been calculated by Monte Carlo simulation with 200 trials.

In Figure 1, the IF is plotted versus SDR for $\rho=0$, where the concerned time-delay interval of each subjamming signal is $1 \mu \mathrm{s}$. The simulation curves show that the approach ensures at least about $20 \mathrm{~dB}$ performance gain under the conditions where JSR is set to 4,8 , and $12 \mathrm{~dB}$, respectively. It is clear 


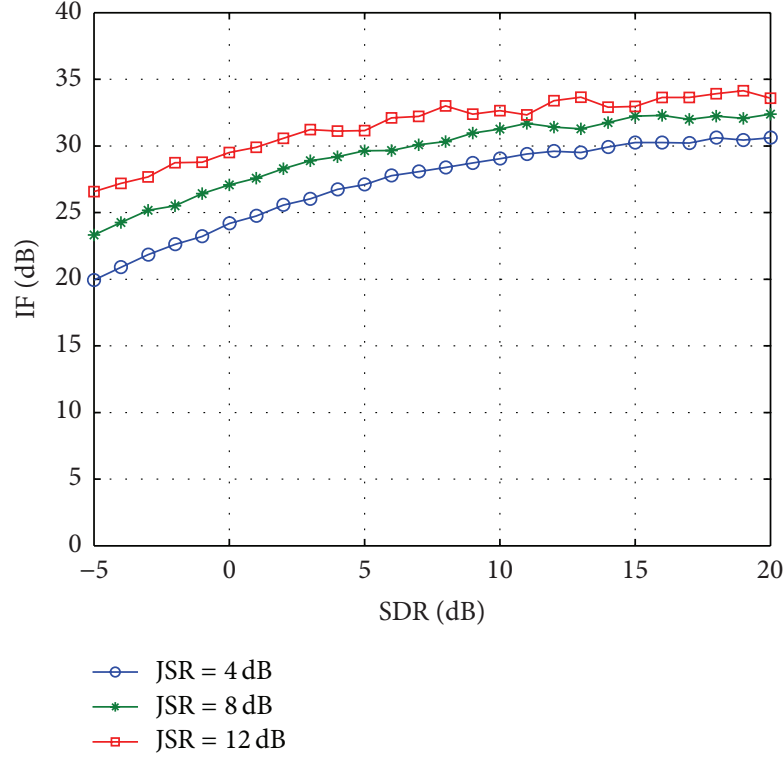

FIGURE 1: Suppression performance of LFM signal, $\rho=0$.

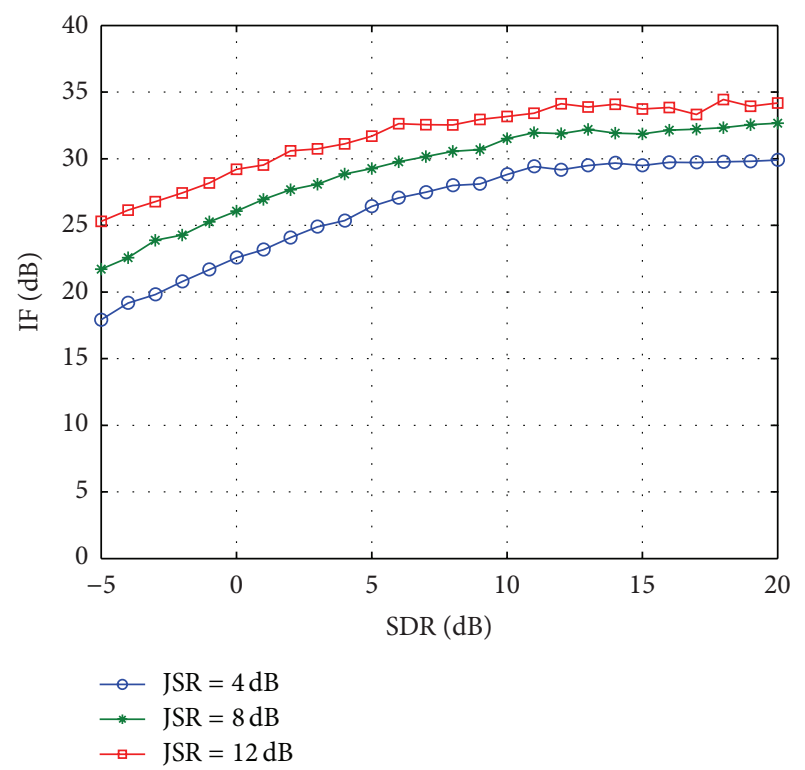

FIGURE 2: Suppression performance of LFM signal, $\rho=0.9$.

that each IF curve approximates to a constant value which exceeds $30 \mathrm{~dB}$ with the increase of SDR under the simulation conditions. Similarly, the performance IF is given in Figure 2, where the simulation parameters remain unchanged except for $\rho=0.9$. As a result there is a slight performance reduction in low SDR for this condition, but it keeps above $18 \mathrm{~dB}$. As SDR increases, the performance difference in the two cases gradually decreases, and the IF also exceeds $30 \mathrm{~dB}$ in high SDR. It is evident that the algorithm performs better in white Gaussian disturbance.

The cases of jammer with respect to a biphase coded radar are analyzed in Figure $3(\rho=0)$ and Figure $4(\rho=0.9)$, where the time-delay interval is $2 \mu$ s and JSR is also set to 4,8 ,

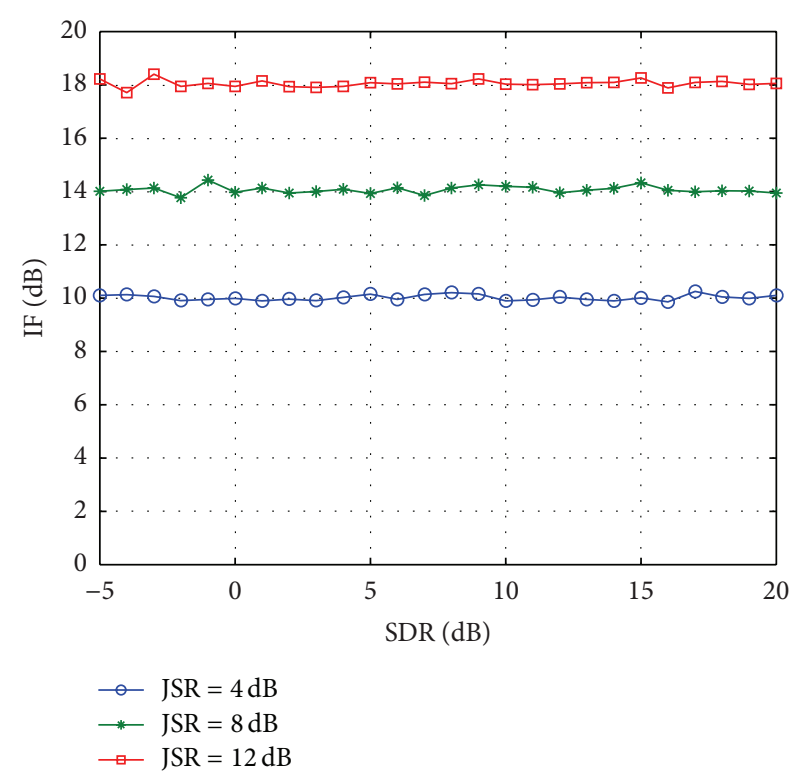

FIGURE 3: Suppression performance of biphase coded signal, $\rho=0$.

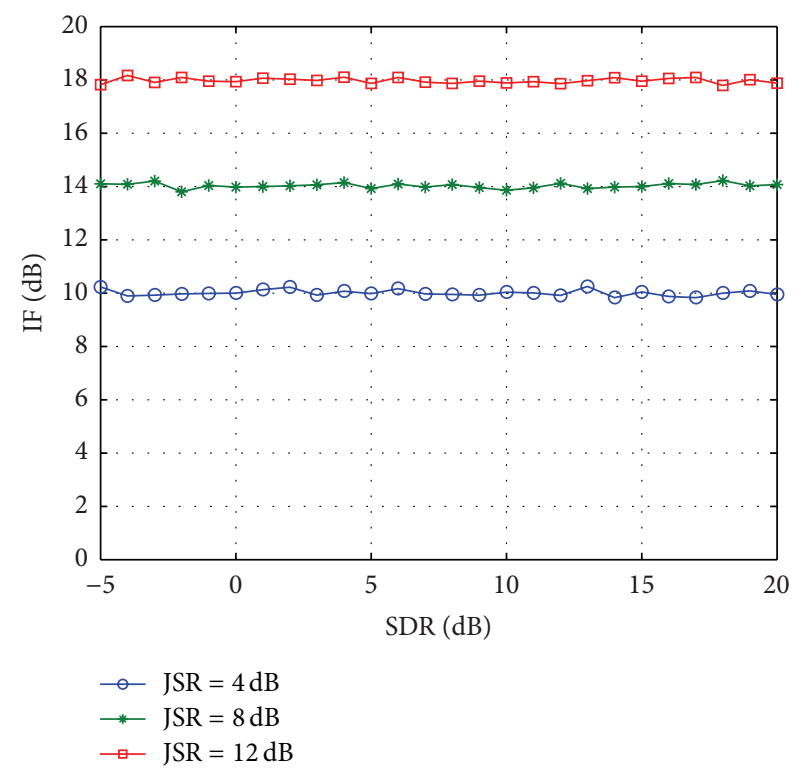

FIGURE 4: Suppression performance of biphase coded signal, $\rho=$ 0.9 .

and $12 \mathrm{~dB}$, respectively. The improvement factor IF has been calculated by Monte Carlo simulation with 200 trials.

As shown in Figures 3 and 4, the improvement factor IF in Gaussian white disturbance and correlated disturbance is approximately the same. Moreover, by investigating the IF, it can be concluded that the SDR has little impact on the final performance curves under the considered conditions.

In simulations, the JSR is set to 4,8 , and $12 \mathrm{~dB}$ due to the fact that a whole radar pulse is stored and repeated; namely, the processing gain can also be achieved by a jammer. 


\section{Conclusions}

In this paper, we investigated the problem of suppressing the so-called repeat-intensive false targets jammer by processing the data during the ToT. By applying the STBC temporally, we can use this technique in surveillance radar systems without multiple antennas. Therefore, a STBC pulse sequence should be used successively in such a system, and if a multiantenna radar system is assumed, namely, if a true STBC can be implemented in different antenna and time slots, a better consequence could be achieved as expected. A WMF is utilized to handle the received radar signals in the presence of jammers with a second-order STBC pulse diversity signal. We analyzed and compared the performance of the proposed algorithm both in white and correlated Gaussian disturbance. This EP technique can be used effectively to cancel a repeatintensive false targets jammer both for LFM and phase-coded radars.

\section{Acknowledgments}

This work was supported by the Key Scientific Research Fund Project of Xihua University (no. Z1220936) and the Open Research Fund of Key Laboratory of Sichuan Signal and Information Processing, Xihua University (no. SZJJ2012013).

\section{References}

[1] P. E. Pace, D. J. Fouts, S. Ekestorm, and C. Karow, "Digital falsetarget image synthesiser for countering ISAR," IEE Proceedings, vol. 149, no. 5, pp. 248-257, 2002.

[2] J. Schuerger and D. Garmatyuk, "Deception jamming modeling in radar sensor networks," in Proceedings of the IEEE Military Communications Conference (MILCOM '08), pp. 1-7, San Diego, Calif, USA, November 2008.

[3] S. D. Berger, "Digital radio frequency memory linear range gate stealer spectrum," IEEE Transactions on Aerospace and Electronic Systems, vol. 39, no. 2, pp. 725-735, 2003.

[4] M. Soumekh, "SAR-ECCM using phase-perturbed LFM chirp signals and DRFM repeat jammer penalization," IEEE Transactions on Aerospace and Electronic Systems, vol. 42, no. 1, pp. 191205, 2006.

[5] J. Akhtar, "Orthogonal block coded ECCM schemes against repeat radar jammers," IEEE Transactions on Aerospace and Electronic Systems, vol. 45, no. 3, pp. 1218-1226, 2009.

[6] Y. Seliktar, D. B. Williams, and E. J. Holder, "Beam-augmented STAP for joint clutter and jammer multipath mitigation," IEE Proceedings, vol. 147, no. 5, pp. 225-232, 2000.

[7] D. J. Rabideau, "Clutter and jammer multipath cancellation in airborne adaptive radar," IEEE Transactions on Aerospace and Electronic Systems, vol. 36, no. 2, pp. 565-583, 2000.

[8] P. M. Corbell, M. A. Temple, T. B. Hale, W. P. Baker, and M. Rangaswamy, "Performance improvement using interpulse pattern diversity with space-time adaptive processing," in Proceedings of the IEEE International Radar Conference, pp. 55-60, Arlington, Tex, USA, May 2005.

[9] L. Lightfoot and T. Li, "Jamming mitigation using space-time coded collision-free frequency hopping," in Proceedings of the IEEE International Conference on Acoustics, Speech, and Signal
Processing (ICASSP '09), pp. 2717-2720, Taipei, China, April 2009.

[10] F. Gini and A. Farina, "Vector subspace detection in compoundGaussian clutter-part I: survey and new results," IEEE Transactions on Aerospace and Electronic Systems, vol. 38, no. 4, pp. 1295-1323, 2002.

[11] C. Xu and K. S. Kwak, "On decoding algorithm and performance of space-time block codes," IEEE Transactions on Wireless Communications, vol. 4, no. 3, pp. 825-829, 2005.

[12] S. M. Alamouti, "A simple transmit diversity technique for wireless communications," IEEE Journal on Selected Areas in Communications, vol. 16, no. 8, pp. 1451-1458, 1998.

[13] V. Tarokh, A. Naguib, N. Seshadri, and A. R. Calderbank, "Combined array processing and space-time coding," IEEE Transactions on Information Theory, vol. 45, no. 4, pp. 1121-1128, 1999.

[14] V. Tarokh, H. Jafarkhani, and A. R. Calderbank, "Spacetime block coding for wireless communications: performance results," IEEE Journal on Selected Areas in Communications, vol. 17, no. 3, pp. 451-460, 1999. 

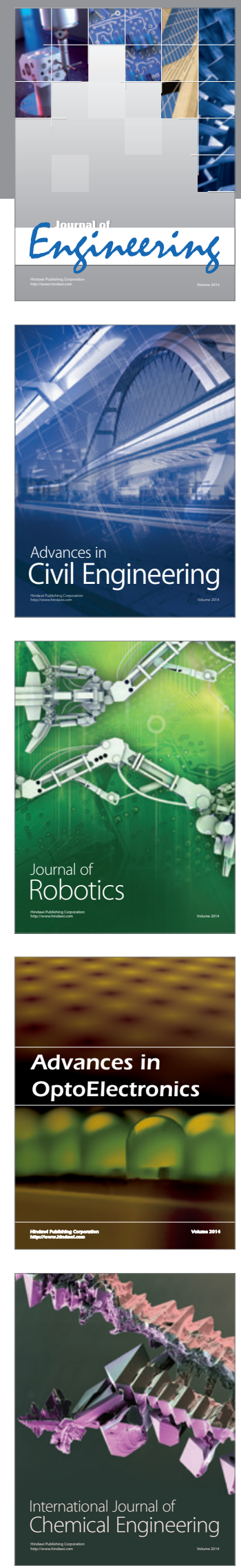

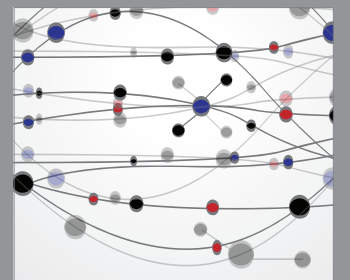

The Scientific World Journal
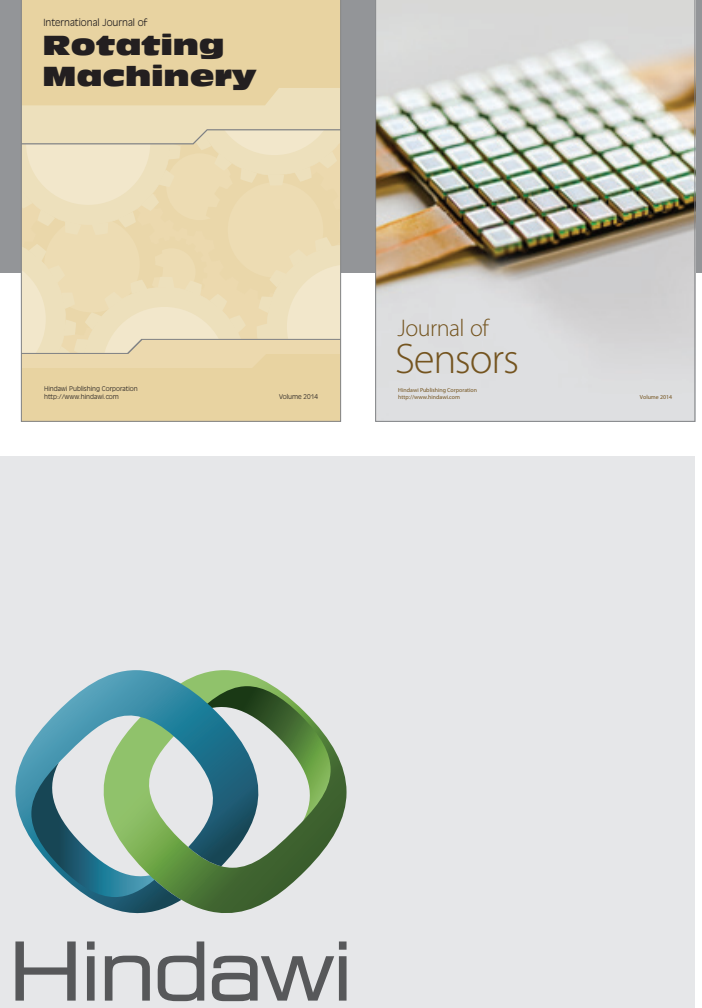

Submit your manuscripts at http://www.hindawi.com
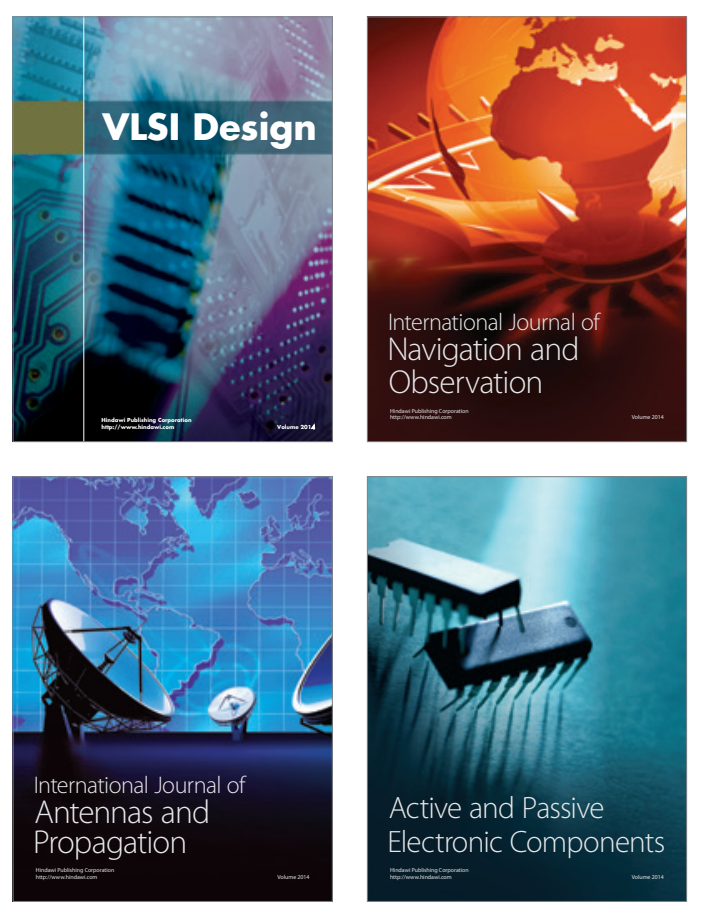
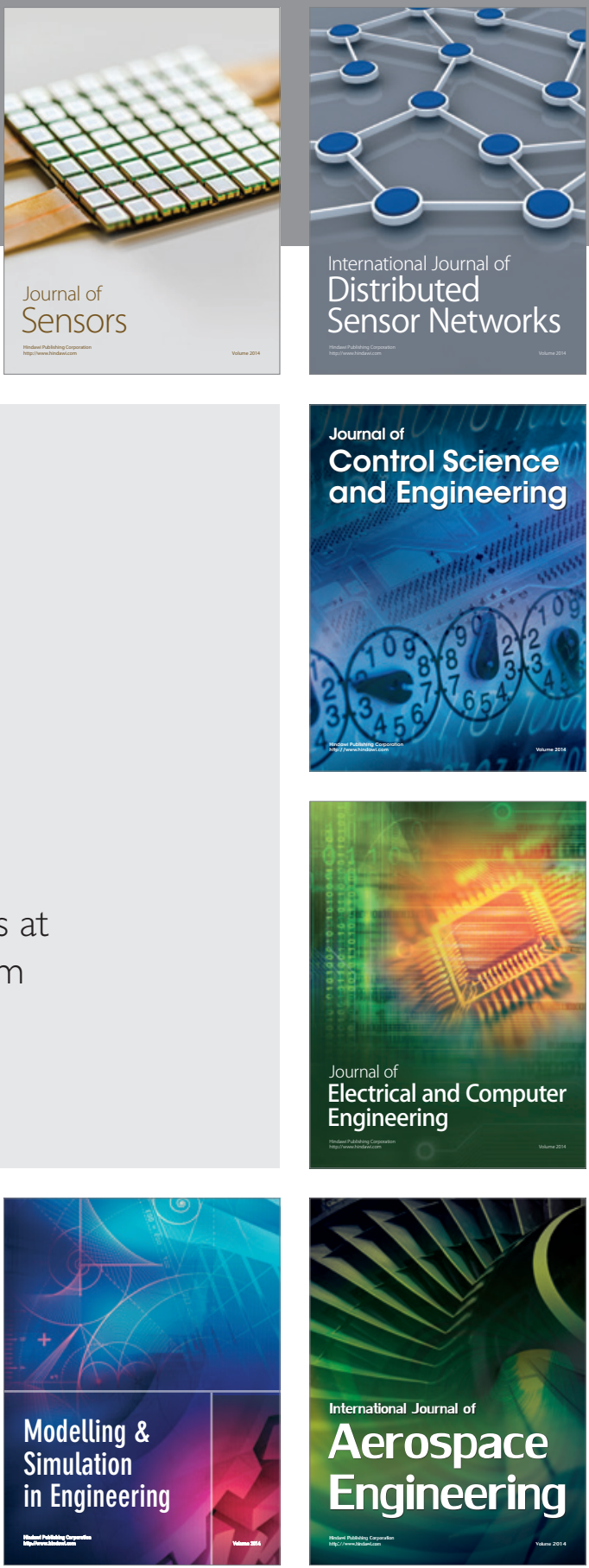

Journal of

Control Science

and Engineering
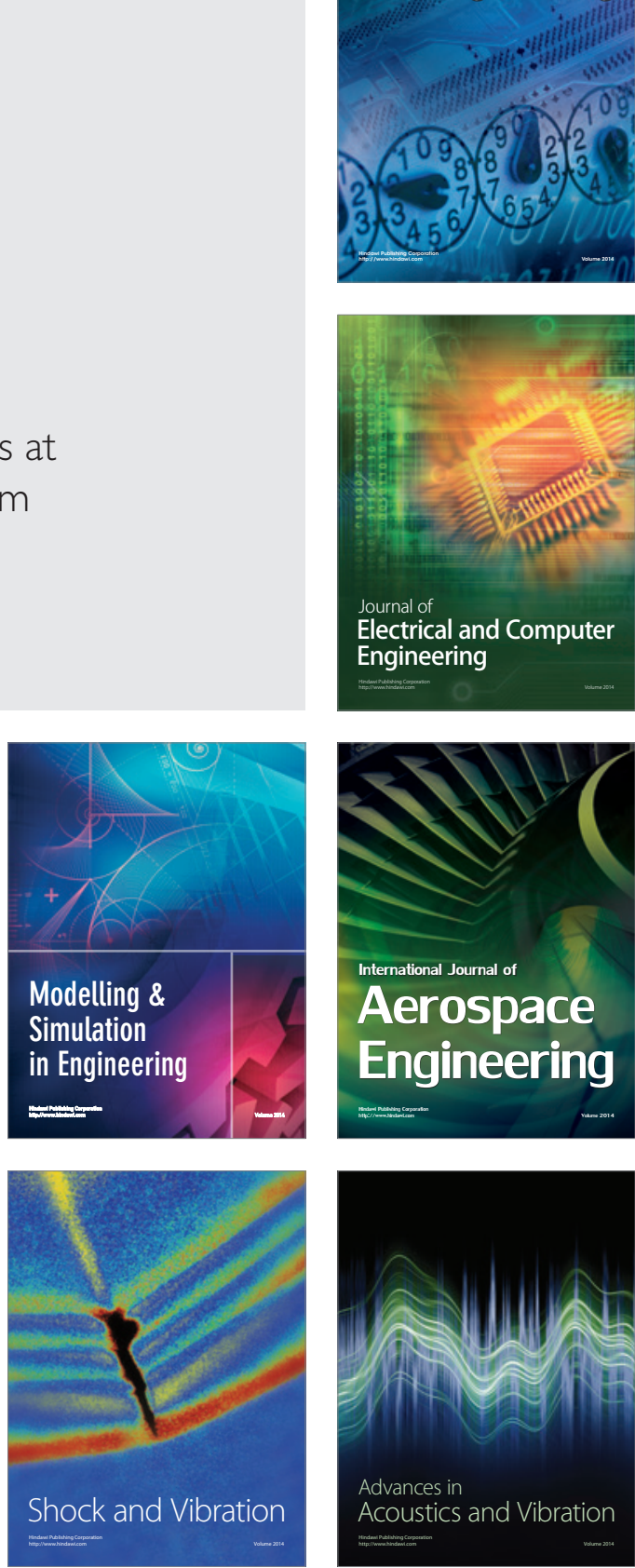